\title{
Laboreal
}

Volume $3 \mathrm{~N}^{\circ} 1$ | 2007

Varia

\section{Error, autonomía y gestión individual y colectiva de la producción}

Erro, autonomia e gestão individual e colectiva da produção

Erreur, autonomie et gestion individuelle et collective de la production

Error, autonomy and individual and collective management of production

\section{Catarina Silva}

\section{(2) OpenEdition}

Journals

\section{Edición electrónica}

URL: http://journals.openedition.org/laboreal/12898

DOI: 10.4000/laboreal. 12898

ISSN: 1646-5237

\section{Editor}

Universidade do Porto

\section{Referencia electrónica}

Catarina Silva, «Error, autonomía y gestión individual y colectiva de la producción », Laboreal [En línea], Volume $3 \mathrm{~N}^{0} 1$ | 2007, Publicado el 01 julio 2007, consultado el 24 septiembre 2020. URL : http:// journals.openedition.org/laboreal/12898; DOI : https://doi.org/10.4000/laboreal.12898

Este documento fue generado automáticamente el 24 septiembre 2020

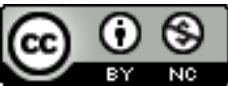

Laboreal está licenciado com uma Licença Creative Commons - Atribuição-NãoComercial 4.0 Internacional. 


\section{Error, autonomía y gestión individual y colectiva de la producción}

Erro, autonomia e gestão individual e colectiva da produção

Erreur, autonomie et gestion individuelle et collective de la production

Error, autonomy and individual and collective management of production

\section{Catarina Silva}

\section{REFERENCIA}

Silva, C. (2006). Erro, autonomia e gestão individual e colectiva da produção : interrogar as práticas orgnizacionais de prevenção do erro. Tese de Doutoramento, Universidade Técnica de Lisboa, Lisboa.

\section{NOTA DEL EDITOR}

Manuscrito recibido en : marzo/2007

Aceptado tras peritage en : junio/2007

\section{Punto de partida}

1 Situamos el punto de partida de este trabajo en las reflexiones suscitadas por los resultados obtenidos en la investigación realizada (Silva \& Lacomblez, 1998), algunos años antes, en una industria de montaje de cableados para vehículos donde cuantificamos la ocurrencia, la detección y la recuperación del error por las propias operadoras. 
2 El estudio que ahora presentamos fue realizado en una empresa que se dedica al montaje, entre otros productos, de radios para la industria automóvil, laborando en tres horarios consecutivos con equipos fijos.

El análisis preliminar de la organización y actividad de trabajo nos mostró que en el contexto de montaje de radios para vehículos las operadoras no estaban implicadas en la gestión de la calidad en los mismos moldes que observamos en el montaje de cableados, debido tanto a la implementación de sistemas de detección de error en los puestos de trabajo existentes en la línea de montaje, como a la adopción de reglas explícitas, separando, por equipos distintos, la actividad de montaje de reparación.

Así, asumimos como objetivo de la investigación analizar las consecuencias de las opciones de organización del trabajo sobre el error en una línea de montaje final de radios para vehículos, recurriendo a un planteamiento longitudinal retrospectiva de la ocurrencia de error.

5 Procuramos comprender, tanto el alcance de la concepción del control de calidad, que no aleja la ocurrencia del error, pero inhibe la gestión del error en tiempo real por las operadoras de montaje final, como el papel de las dinámicas de regulación del proceso de trabajo, individuales y colectivas, indispensables a la gestión de la producción, bajo el efecto de una organización que consideramos discrecional,

Del punto de vista teórico, nos apoyamos en los trabajos de Maggi (2003) ; de Terssac $(1992 ; 2002)$ de Terssac y Dubois (1992) y de Reynaud $(1999,2004)$, los cuales nos permitieron una interpretación de la actividad de trabajo considerando los cambios organizacionales que hoy se imponen al modelo tayloristafordista.

\section{Una organización postfordista}

7 ¿Qué "nueva" organización del trabajo estaba entonces en causa? Intentamos respuestas a cuestiones que espejan las trayectorias de cambio del modelo clásico "Scientific Management" (taylorista-fordista) y que se imponen, hoy, a los sistemas productivos : (1) ¿Cómo se gestiona la incertidumbre de los pedidos de los clientes? (2) ¿Cómo se procesan las relaciones clienteproveedor en el interior de la empresa? (3) ¿Cómo es alcanzada la reducción de los plazos comerciales? (4) ¿Cómo se gestiona la enorme variedad de los productos? (5) ¿Cómo se hace y coordina el control cuantitativo y cualitativo de la producción?

Constamos estar en presencia de una organización del trabajo dicha póstfordista, pues ejerce igualmente la racionalización del trabajo, pero basada, de acuerdo con Maggi, Terssac y Dubois, en espacios de acción discrecionales, es decir, que solicita de los actores iniciativa, innovación, responsabilidad, decisión y reconoce (por lo menos en parte) sus competencias, pero en un marco de dependencia determinado superiormente.

9 El uso de la discreción - y no cedencia de autonomía, dado que esta supone independencia de los sujetos en la conducción de los procesos de acción - se hace imprescindible para asegurar la producción en condiciones de incertidumbre permanente. 


\section{La regulación del proceso de trabajo}

10 La regulación es un proceso central para las organizaciones que privilegian la discreción como modo de hacer frente a la incertidumbre con que se confrontan permanentemente. Este presupuesto nos llevó a analizar la actividad de trabajo de montaje de radios para vehículos, procurando respuestas a las cuestiones : (1) ¿Cuáles son las obligaciones implícitas en una tarea discrecional ? (2) ¿Qué actividad, individual y colectiva responde a una tarea de esta naturaleza? (3) ¿Qué proceso de regulación social impone este tipo de tarea?

11 Dos evidencias emergieron. Del punto de vista conceptual creímos haber mostrado la proficuidad para la ergonomía de movilizar para lectura de lo real del trabajo otros marcos teóricos, en este caso concreto, de la sociología del trabajo protagonizada por Terssac y Reynaud. Al introducir en nuestro análisis los diferentes sistemas de reglas que cohabitan y se combinan en los procesos de trabajo, conseguimos superar la visión "taylorista" de la ergonomía de confrontar la tarea (reglas de control) con la actividad (reglas autónomas) y hacer emerger los procesos de regulación conjunta (reglas efectivas). Del punto de vista empírico, los datos nos mostraron qué compleja es la regulación del proceso de trabajo, tanto del punto de vista individual como colectivo ; como esa regulación asentaba inevitablemente en iniciativas, en tomas puntuales de "autonomía", para hacer frente a los constreñimientos que cotidianamente se atraviesan en el proceso de trabajo; en fin, como la regulación tenía subyacente procesos de negociación, no siempre claramente explícitos, entre diferentes estructuras de decisión - vertical o horizontal pero absolutamente esenciales para el alcance de los objetivos de la empresa.

\section{Producción, error y organización del trabajo}

\subsection{Cronología de acontecimientos}

12 El conocimiento del funcionamiento del sistema productivo nos llevó a cuestionar la eficacia de las opciones de organización del trabajo en la ocurrencia de error. Siéndonos apenas posible obtener datos retrospectivos de error (registros archivados provenientes de la estación de reparación), decidimos pesquisar los acontecimientos ocurridos en la línea de montaje relativos :

- a la forma de gestión de los recursos humanos (movilidad);

- a las variaciones del planeamiento de la producción (volumen y diversidad); (3) a la transición entre productos (descontinuados y nuevos); (4) a los cambios del proceso de montaje (estructurales y funcionales), teniendo en vista elaborar una cronología que nos ayudase a interpretar los resultados del tratamiento de los datos del error, considerando el conocimiento adquirido sobre la situación de trabajo. Dieciocho meses constituyeron nuestro período de análisis retrospectivo.

\section{2. ¿Qué nos dicen los errores?}

Del tratamiento de nuestros datos, el error se manifestó como una de las consecuencias de opciones de gestión de recursos humanos basada en criterios, esencialmente, económicos y comerciales. Creemos que mejores resultados serian obtenidos si hubiese 
una apuesta efectiva en la estabilización (y no en la movilidad) de los recursos humanos - en concreto del tercer horario (de la noche) y un reconocimiento del lugar del saberhacer, además de la mera ejecución de operaciones de montaje. Vimos cuanto la movilidad de los recursos humanos puede comprometer la construcción de la representación común, esencial cuando el apelo al colectivo es más vehemente aumento del volumen de producción, transiciones entre productos, disfuncionamientos de los equipos de la línea etc. - viabilizando mayores fracasos. La racionalización de los recursos humanos, practicada con el objetivo de alcanzar ganancias económicas, acarrea costos añadidos en la producción, derivados de los errores humanos.

Verificamos que a pesar de una concepción de la organización del trabajo visando la prevención y la detección precoz del error, el error seguía ocurriendo. Las lagunas de esta concepción eran superadas por la dinámica colectiva de gestión de la actividad, que vimos ser practicada por el equipo del primer horario, pues permitía un mejor control del tiempo, de la producción y de sus resultados, en términos de error. En esta línea de montaje, la no imposición de una cadencia tenía subyacente la posibilidad de hacer uso discrecional del tiempo, con el objetivo de alcanzar la producción, y no de humanizar el trabajo.

Constatamos, también, que la gestión de la variabilidad de producción y luego del error salió beneficiada por las transformaciones que fueron siendo introducidas en la disposición de la estructura física de la línea de montaje.

El análisis detallado de los procedimientos empleados reveló redefiniciones visando la fiabilidad del producto. Observamos la práctica de operaciones suplementares de prevención, con éxito, a determinados aspectos del producto, para los cuales no existía otro medio de control. Constatamos, como la realización de pequeñas reparaciones (jclandestinas!), acababan por ser objeto de negociación con la jerarquía: se intercambia el reconocimiento de la competencia para hacer recuperaciones por la garantía de cumplir el planeamiento. Confirmamos el envolvimiento de las operadoras en la gestión del error, a pesar de no estar contemplado en la organización formal.

Presenciamos como la acción colectiva va también en el sentido de procurar la regulación de la carga de trabajo en el seno del equipo, de promover la construcción de la competencia individual, pero también, colectiva, de facilitar la gestión de las situaciones críticas de trabajo, así como de prevenir conflictos, etc. Por eso, podemos afirmar que el error estaba también en el centro de procesos de regulación social.

\section{5. ¿Concepción preventiva del error o aprender con el cotidiano?}

18 Este trabajo incita a repensar si la apuesta debe de ser efectivamente en el sentido de una concepción preventiva de los errores o si debemos aprender con las prácticas del cotidiano. Vimos como esta concepción preventiva del error olvida: 1) las competencias, las iniciativas, muchas veces individuales pero cuyo resultado se refleja en el trabajo de los otros ; y 2) que el error es, también, objeto de negociación social y un medio al servicio de la fiabilidad. Nos pareció estar ante una gestión del error dejada a la deriva de la organización informal del trabajo. Más que concentrar esfuerzos buscando en los espacios, que acaban por quedar a la responsabilidad e iniciativa de los operadores, donde él erró, lo que violó, nos parece más interesante ver como, en ese 
mismo espacio, la gestión informal del error y de la producción acaba por emerger. Se trata, pues, de mirar la construcción de reglas propias de acción, que no se deben confundir, en este contexto, con una verdadera autonomía, pero antes considerarse como conquistas puntuales implícitamente negociadas, en el seno de los colectivos y con su jerarquía. Esta gestión informal al confundirse con la actividad de trabajo corriente, nos exige una mirada atenta. Es también preciso superar las interpretaciones de los propios operadores que desvalorizan esas iniciativas individuales, no las reconociendo como señales fundamentales de su competencia. El análisis de las actividades de trabajo, más que focalizarse en los acontecimientos excepcionales, debe pues considerar las prácticas del cotidiano. De este punto de vista será interesante retener la perspectiva de la teoría del actuar organizacional (Maggi, 2003) que opta por hablar en ajustes continuados, necesarios e inevitables durante el desarrollar del proceso de acción en vez de error.

Realzamos el interés y la necesidad de repensar formulas organizacionales que reconozcan esas prácticas del cotidiano como verdaderas actividades profesionales, abriendo camino a un planteamiento que dará, ciertamente, mayor visibilidad a la participación activa de los operadores en la gestión de su proceso de trabajo.

\section{BIBLIOGRAFÍA}

Terssac, G. de (1992). Autonomie dans le travail. Paris : PUF.

Terssac, G. de \& Dubois, P. (Eds.). (1992). Les nouvelles rationalisations de la production.

Toulouse : Cépadues Editions.

Terssac, G. de (Ed.). (2002). Le travail : une aventure collective. Toulouse : Editions Octares.

Maggi, B. (2003). De l'agir organisationnel. Un point de vue sur le travail, le bien-être, l'apprentissage. Toulouse : Octares Editions.

Reynaud, J.-D. (1999). Le conflit, la négociation et la règle (2 $2^{\mathrm{a}}$ ed.). Toulouse : Editions Octares.

Reynaud, J.-D. (2004). Les règles du jeu. L'action collective et la régulation sociale. Paris : Armand Colin.

Silva, C. \& Lacomblez (1998). Quand le contrôle de la qualité vient, toute contrainte de temps étant égale par ailleurs : l'erreur humaine dans le montage de câbles électriques pour véhicules. Paper presented at the XXXIIICongresso da SELF “ Temps et travail », Paris, Setembro, 16-18.

\section{AUTOR}

\section{CATARINA SILVA}

Faculdade de Motricidade Humana Estrada da Costa, Dafundo, 1495 - 688 Lisboa Portugal csilva@fmh.utl.pt 